\title{
SEARCH ENGINE OPTIMIZATION (SEO) APLICADAS EM COMÉRCIO ELETRÔNICO
}

\section{SEARCH ENGINE OPTIMIZATION (SEO) APPLIED TO E- COMMERCE}

\author{
Gustavo Camossia \\ Heytor Diniz Teixeirab \\ Cecilio Merlotti Rodas ${ }^{c}$ \\ Rachel Cristina Vesu Alves ${ }^{d}$
}

\begin{abstract}
RESUMO
Objetivo: O presente artigo tem como objetivo apresentar a experiência de um comércio eletrônico durante a implementação de algumas técnicas de Search Engine Optimization (SEO). Metodologia: Trata-se de um estudo qualitativo exploratório e de natureza aplicada, sendo o seu escopo a observação direta, sem participantes, e tendo como método a netnografia para coleta e análise de dados. Resultados: Verificou-se que a utilização das técnicas de Search Engine Optimization na construção dos sites de comércio eletrônico é eficiente e adequada para otimização desses ambientes. Conclusões: Conclui-se que as técnicas de Search Engine Optimization e seus elementos, devidamente aplicados, auxiliam na organização, representação e recuperação da informação e tornam o site mais visível nos mecanismos de busca.
\end{abstract}

Descritores: Search Engine Optimization. Comércio Eletrônico. Recuperação da Informação. Web. Mecanismos de Busca.

\section{INTRODUÇÃO}

As práticas de recuperação, acesso e uso de informações evoluíram significativamente desde o advento dos mecanismos de busca baseados na

\footnotetext{
a Mestrando em Ciência da Informação pela Universidade Estadual Paulista (UNESP). Email: gustavo.camossi@unesp.br

b Mestrando em Ciência da Informação pela Universidade Estadual Paulista (UNESP). Email: hd.teixeira@unesp.br

c Doutor em Ciência da Informação pela Universidade Estadual Paulista (UNESP). Email: cecilio.rodas@unesp.br

d Pós-doutorado em Ciência da Informação pela Universidade Estadual Paulista (UNESP). Email: rachel.vesu@unesp.br
} 
Web. Há algum tempo, por exemplo, a procura por informações, que demandaria uma visita à biblioteca, a mediação de um bibliotecário e uma busca por documentos impressos evoluiu com o surgimento da Internet e dos mecanismos de busca.

Atualmente, os mecanismos de busca, como um novo canal de recuperação da informação, passaram a fornecer acesso rápido a uma grande quantidade de informações, permitindo assim uma redução significativa no tempo da busca. A Internet Live Stats diz que o Google processa mais de 40.000 consultas de pesquisa a cada segundo em média, o que se traduz em mais de 3,5 bilhões de pesquisas por dia e 1,2 trilhão de pesquisas por ano em todo 0 mundo.

A Internet como fonte de pesquisa tem permitido o acesso a um grande volume de informação, o que dificulta a recuperação de conteúdos relevantes. Estar presente na Web e tornar suas informações visíveis, tornou-se um grande desafio em meio à quantidade de ambientes digitais e outras fontes existentes. Em busca de solucionar esse cenário, as técnicas de análise documentária, incluindo a indexação, devem ser aplicadas, uma vez que aumentam as possibilidades de visibilidade e encontrabilidade da informação.

O papel principal que os mecanismos de busca agora cumprem na busca e recuperação fortaleceu a importância dos processos de organização e descrição das informações na Web. Esses processos ocorrem no intuito de impulsionar a visibilidade das páginas nos resultados dos mecanismos de pesquisa, em inglês, Search Engine Result Pages (SERPs).

Ledford (2007) definiu o Search Engine Optimization (SEO) como um conjunto de técnicas que, uma vez aplicadas adequadamente, tornarão um Website mais visível e melhor posicionado nos mecanismos de busca. $O$ principal objetivo do SEO é otimizar os sites para que os mecanismos de busca possam encontrá-los, lê-los, avaliá-los e indicá-los, e, desta forma, torná-los mais relevantes.

Portanto, gerar relevância para as páginas dos sites é importante nas SERPs. Sendo assim, as técnicas de SEO permitem que as páginas se tornem rastreáveis por robôs, que, por sua vez, verificam a relevância das informações 
e contribuem para a sua classificação e, consequentemente, em sua posição no ranqueamento dos resultados de uma busca.

Este artigo tem como objetivo geral, discutir a relevância da aplicação das técnicas de SEO para o comércio eletrônico e a experiência de aplicação de SEO em um site de comércio eletrônico. O objetivo específico é mencionar as estratégias adotadas, as ferramentas de monitoramento utilizadas e alguns resultados alcançados até o momento, bem como algumas solicitações de continuidade dos trabalhos realizados e os resultados esperados no longo prazo.

\section{A IMPORTÂNCIA DOS MECANISMOS DE BUSCA PARA A RECUPERAÇÃO DA INFORMAÇÃO NO COMÉRCIO ELETRÔNICO}

Em vista do valor estratégico da informação, à medida que a sociedade se transforma, também mudam as pesquisas, os processos, os métodos e as práticas na Ciência da Informação $(\mathrm{Cl})$. As Tecnologias de Informação e Comunicação (TIC) estão consolidadas no cotidiano das pessoas e tornou a recuperação da informação mais dinâmica, o que desafiou os pesquisadores a compreender esse processo de transformação, principalmente, no campo da $\mathrm{Cl}$, em que se busca elementos para melhorar o tratamento e a organização da informação para facilitar e possibilitar o seu acesso Dentre os meios de comunicação desenvolvidos, a Internet é o que tem maior extensão e aderência mundial, tendo ultrapassado os meios tradicionais, como televisão, rádio e jornais impressos.

No que se refere ao comércio eletrônico (e-commerce), esses sistemas devem prover aos consumidores uma conexão entre as empresas e os seus usuários, apoiar diretamente a troca de informações, eliminar as restrições de tempo e espaço e apoiar os consumidores. Interagir para se adaptar ao comportamento dos consumidores eletrônicos é necessário, pois cada vez mais os consumidores realizam compras no ambiente digital. Portanto, os desenvolvedores precisam se preocupar não apenas com as ferramentas, mas também com o que os consumidores têm a dizer sobre suas experiências no ecommerce (ALBERTIN, 2010). 
É de extrema importância para o e-commerce identificar novas tecnologias, hábitos e produtos que se inserem no contexto da Web, e que estão disponíveis desde a concepção e criação dos artefatos até à sua compra e utilização pelos usuários.

Sendo assim, Albertin (2010) afirma que e-commerce é uma forma de comércio eletrônico que, devido ao alcance da Internet, tem o poder de conectar varejistas em todo o mundo. Caracteriza-se por ser um ambiente repleto de tecnologias de comunicação e informação e visa a concretização dos objetivos de negócio, sendo conhecida pelo seu fácil acesso e baixo custo.

Para Gabriel (2018), a tecnologia digital, aliada ao potencial atual de uso da Internet, permite que os usuários se conectem 24 horas por dia, levando a uma extensão do corpo biológico, passando a utilizar os dispositivos digitais como extensão do seu próprio corpo. Battelle (2005), em suas reflexões, discorre que no futuro próximo a busca ampliará suas origens na Web, tornando-se acessível para todos os dispositivos.

Dito isso, no primeiro trimestre de 2020, a Compre \& Confie, empresa de pesquisa de mercado, realizou levantamento no varejo digital e apresentou um crescimento significativo devido ao COVID-19. Naquela época, as vendas no mesmo período do ano passado chegaram a 20,4 bilhões (NOVAES, 2020).

Segundo Kemp (2021), o número de usuários da Internet no Brasil aumentou para 9,6 milhões (+ 6,4\%) entre 2020 e 2021 e o número de conexões móveis no Brasil em janeiro de 2021 era equivalente a $96,3 \%$ da população total (Figura 1). 
Figura 1 - Crescimento Digital Anual no Brasil

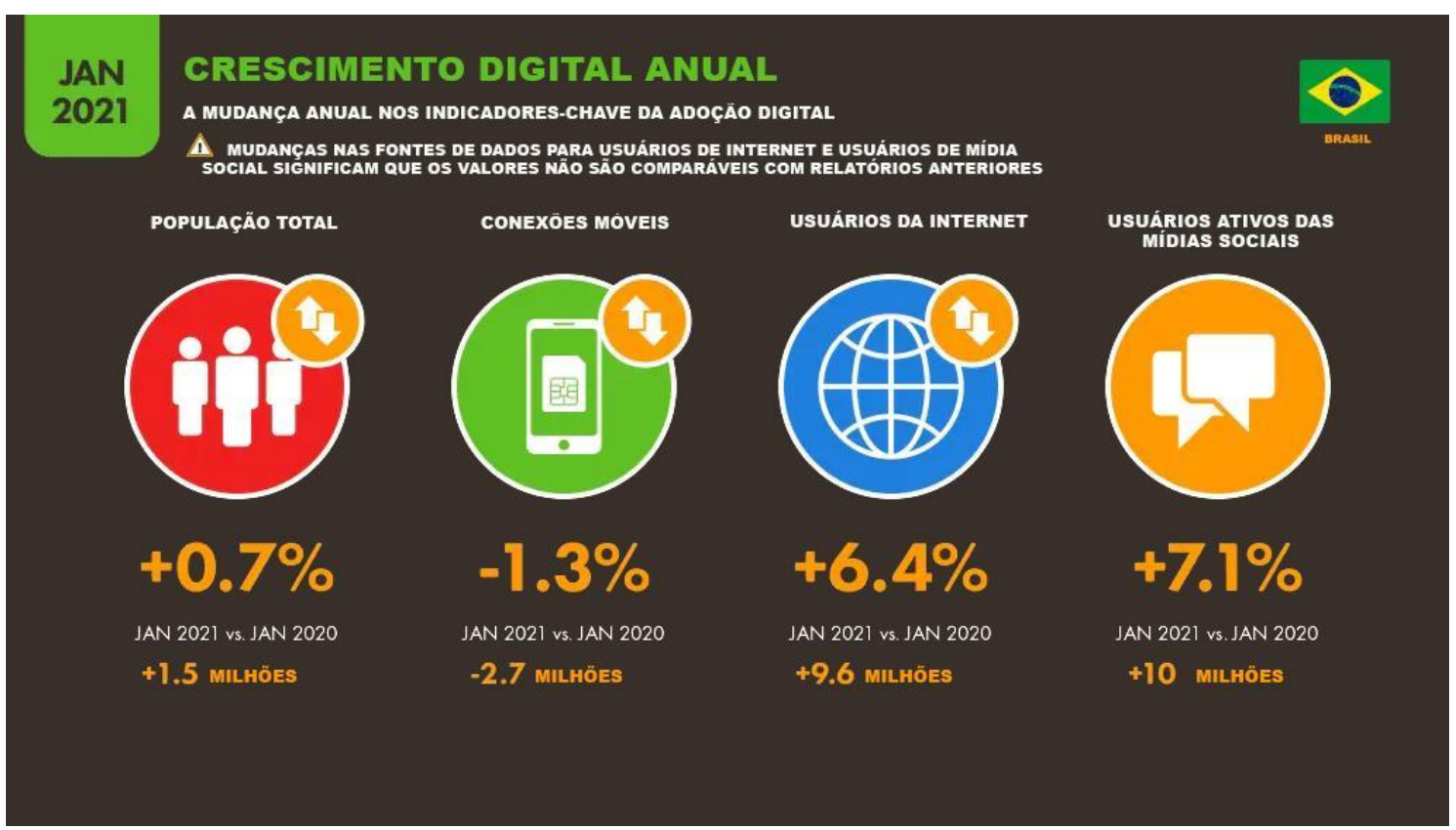

Fonte: Kemp (2021)

Um crescimento expressivo nas compras online foi notado com base na Ebit, uma organização global de medição e análise de dados que apresenta uma visão mais integrada e confiável do mundo dos consumidores e mercados em um site eletrônico. A Figura 2 mostra o faturamento recorde em 5 anos de $e$ commerce no primeiro semestre de 2020.

Figura 2 - Recorde de faturamento no primeiro semestre de 2020

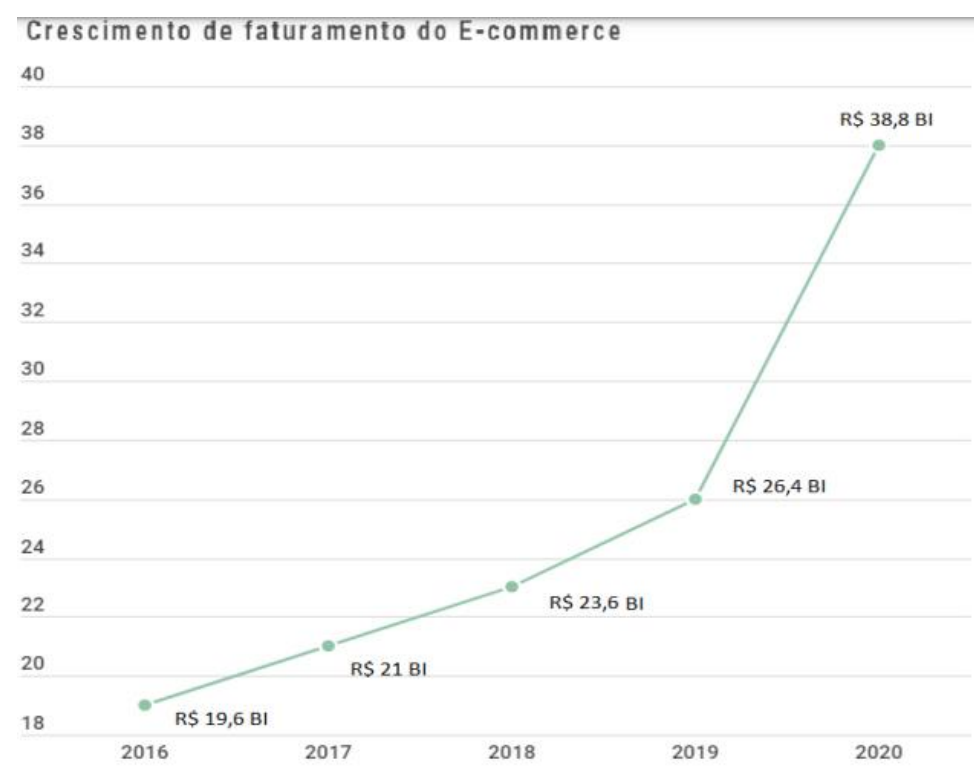

Fonte: Adaptado de Ebit (2020) 
A partir desse cenário, as considerações para a construção e disponibilização de informações em ambientes digitais são fatores que requerem da $\mathrm{Cl}$ e outras áreas como a Ciência da Computação, por exemplo, um olhar crítico sobre a organização das informações na Web, e que se utilizem de meios para melhor analisar e compreender os aspectos que podem influenciar em seu acesso.

Portanto, para tratar a vasta quantidade de informação na Web, se fez necessário desenvolver novas práticas de organização e novas tecnologias para a recuperação. Os mecanismos de busca surgiram para atender a demanda por melhoria no processo de recuperação de informações (CENDÓN, 2001).

Martins e Carvalho Junior (2017) dizem que a principal força sociotécnica por trás de serviços como Google, por exemplo, é sua capacidade de processar uma quantidade única de informações que cobre uma ampla variedade de interesses e expressões humanas. Para fazer isso, eles usam critérios de relevância social, segmentando por relevância e de acordo com interesses específicos, apresentando resultados satisfatórios, destacando-se por serem facilmente utilizados pelos usuários. Para propiciar a encontrabilidade da informação, é necessário possuir relevância na Web, fator importante a se considerar para a organização de informações na atualidade.

Um estudo realizado por Bohórquez (2012) aborda o uso das técnicas de SEO em sites de bibliotecas. Essas técnicas podem criar diversas maneiras para salientar os conteúdos, produtos e serviços oferecidos na Internet, principalmente, por meio dos mecanismos de busca, para que os usuários obtenham informações eficientes de maneira simples e clara.

Com base no que foi revelado, pode-se observar na figura 3 que um comércio eletrônico possui diversas motivações para aplicar técnicas de SEO, principalmente, ao considerar a combinação dos seus recursos com os dos mecanismos de busca. O poder de conectividade e alcance desses sistemas de recuperação da Web oferecem uma entrega de informações confiáveis para os usuários. 
Figura 3 - Principais motivos para aplicar o SEO
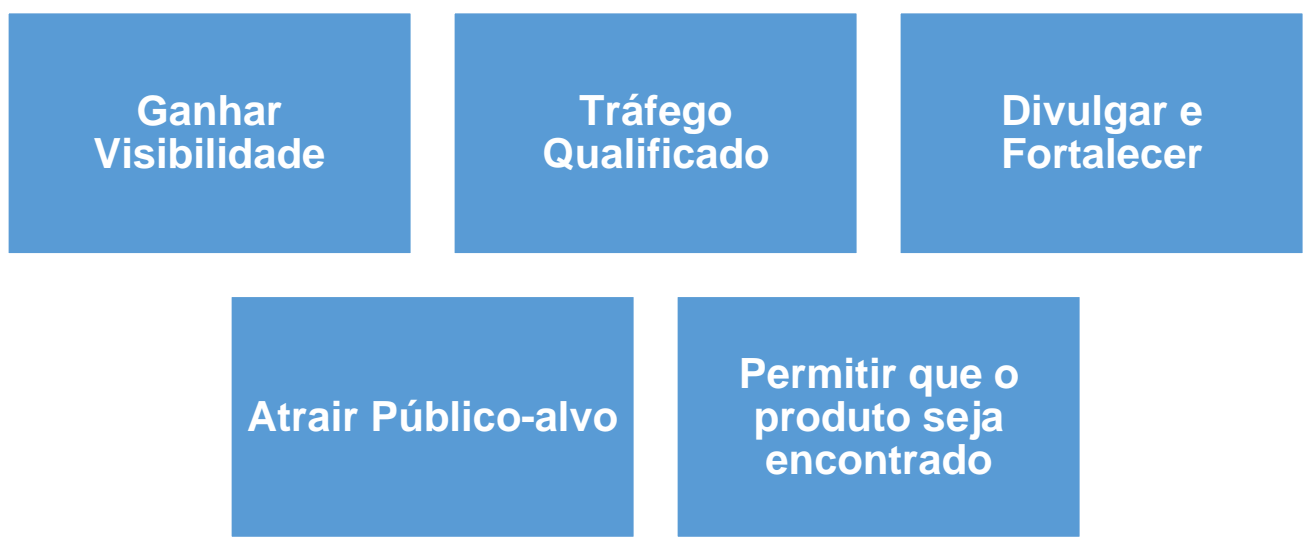

\section{Permitir que o}

produto seja

encontrado

Fonte: Elaborado pelos autores (2021)

É necessário compreender as características e potencialidades do ambiente Web para agregar produtos e / ou serviços em ambientes digitais com características atuais e especiais. Consequentemente, o profissional da informação deve adquirir novas funções, habilidades e conhecimento contínuo para atender às demandas desses ambientes em constante mudança.

\section{SEARCH ENGINE OPTIMIZATION}

A busca por informação não é algo recente, visto que os usuários necessitam deste bem, de valor estratégico para a tomada de decisões. Entretanto, no decorrer dos anos, a maneira em que a busca por informações evoluiu e, em razão dos avanços tecnológicos e da massificação das TIC, ampliaram-se os canais de acesso. Sendo assim, a informação tornou-se mais presente no cotidiano dos usuários, influenciando o consumo, os hábitos e 0 estilo de vida das pessoas.

Atualmente, as buscas nos ambientes digitais são realizadas por meio de search engines, termo traduzido para o português como "mecanismos de busca" ou "buscador". Search engines é um sistema de recuperação de informações que tem como principal objetivo auxiliar na busca de informações armazenadas em ambientes computacionais (GABRIEL; KISO, 2020). 
Esses mecanismos de busca foram criados para facilitar o acesso à informação, devido à crescente dificuldade de encontrá-las na Web, em decorrência do aumento gradativo das informações (TU; HSIANG, 1998). Portanto, no contexto do comércio eletrônico, a maneira de possibilitar um melhor posicionamento nos resultados de busca dos conteúdos informacionais é empregando as técnicas de SEO em suas páginas.

O SEO é um conjunto de técnicas que, uma vez aplicadas adequadamente, pretende tornar um site mais visível e melhor posicionado na lista de resultados de um mecanismo de busca. O principal objetivo do SEO, portanto, é otimizar os sites para que possam ser encontrados, lidos, avaliados e indexados (LEDFORD, 2007).

Adicionalmente, Ledford (2007) considera que o SEO é o processo a seguir para melhorar a visibilidade de um site nos mecanismos de busca. A otimização de sites para mecanismos de busca está, geralmente, ligada a pequenas modificações em zonas chave desses sites.

O SEO pode ser classificado em duas categorias: SEO on-page e SEO off-page. O SEO on-page está ligado à otimização do conteúdo do Website para tentar combinar a busca de algoritmos dos mecanismos de busca, que é considerada o foco deste artigo. Já o SEO off-page está relacionado ao ganho de links e tráfego para seu site a partir de outros domínios. Estes diferentes mecanismos combinados determinam a classificação e o valor do site quando se trata de visibilidade nos mecanismos de busca. Enquanto ambos são essenciais para um Web site de boa qualidade e bem classificado, SEO off-page possui uma influência no ranking do mecanismo de busca, mas deve sempre ser construído sobre uma constante otimização na página (ZLATIN, 2017).

Essas técnicas, quando combinadas, são capazes de melhorar significativamente a eficiência da pesquisa e a maneira como os usuários encontram e acessam as informações nos mecanismos de busca. Vale salientar que essas técnicas são aplicadas em etapas para verificar se houve melhorias nas páginas e, assim, dar continuidade na aplicação das técnicas. 


\section{PROCEDIMENTOS METODOLÓGICOS}

Esta pesquisa pode ser classificada como qualitativa, exploratória e de natureza aplicada e seu escopo é a observação direta sem participantes e o método da netnografia para coleta e análise de dados. A netnografia é um ramo da etnografia que estuda a tecnologia e o comportamento de grupos sociais na Internet (ALMEIDA; SOUSA; OLIVEIRA, 2018), podendo também ser utilizada para análise de sites.

O universo de estudo constitui-se de um site de comércio eletrônico, que por motivos éticos da pesquisa não será identificado, e, por meio de uma observação direta não participante, buscou-se analisar os seguintes elementos de SEO: Heading tags, Meta tags, Atributos alt, Sitemaps, e Enriquecimento do conteúdo informacional.

Dito isso, foram utilizadas duas ferramentas de levantamento de dados que permitiram analisar a otimização do site: Google Webmaster Tools ${ }^{5} \mathrm{e}$ Google Analytics ${ }^{6}$. Os dados foram levantados no período de junho de 2020 a dezembro de 2020 e constituem a base de análise para discussão dos resultados deste artigo.

A utilização da ferramenta Google Webmaster Tools é a primeira etapa após a implementação de técnicas de SEO. Com esta ferramenta é possível informar ao Google que existe um site e deve ser indexado. O serviço Google Webmaster Tools é um serviço gratuito para Webmasters e usuários do Google e permite realizar uma análise detalhada da indexação de páginas da Web e obter informações sobre seu desempenho no mecanismo de busca Google.

Para a aplicação das técnicas de SEO no site, foi preciso realizar a adequação dos Heading tags, ou "etiquetas de cabeçalho", tais como H1, H2, H3, etc. Conforme afirma (LEDFORD, 2007), as Heading tags são recursos de HTML utilizados para destacar os elementos mais importantes de uma página, como o título e os subtítulos.

Nesse sentido, foram realizadas as inserções das metatags, pois

\footnotetext{
${ }^{5} \mathrm{https} / / /$ search.google.com/search-console.

${ }^{6}$ https://analytics.google.com
} 
identificam o título (tag title), palavras-chave (tag meta keywords) e descrevem o site para os mecanismos de busca (tag meta description). Desse modo, considerando esses aspectos, promoveu-se o preenchimento das metatags de título - a meta title, de descrição - a meta description e as palavras-chave - as keywords. Ainda, no que compreende as tags, também foram promovidos os cadastramentos de atributos alt em imagens do site, que tem como objetivo de fornecer um texto alternativo à imagem, caso ela não seja carregada.

Outro fator importante que se presta atenção ao aumento da indexação de páginas do site é o estudo dos sitemaps. O sitemaps é um elemento que contribui diretamente com os mecanismos de busca para a indexação das páginas do site, especialmente para que os robôs entendam sua estrutura, arquitetura e navegação de páginas e partes (LEDFORD, 2007). Através desta análise foram descobertos diversos problemas que surgiram no sitemaps, após esta, o sitemaps foi configurado para estar de acordo com a situação real das páginas do site.

O enriquecimento da descrição de conteúdo nas páginas do site também é uma etapa importante, pois quanto mais detalhada for a descrição e a representação de produtos e serviços das páginas, maior a probabilidade de serem observadas por robôs, além de serem indexadas e aparecerem no SERP. A organização e a relevância do conteúdo da informação nas páginas são mais importantes do que qualquer componente técnico de SEO.

\section{RESULTADOS: APRESENTAÇÃO E DISCUSSÃO}

Os dados apontam que o site deve sempre se dedicar para manter atualizada frente às técnicas de SEO, uma vez que a maior parte dos acessos obtidos são originados por esses mecanismos. Ao apurar somente o período de dedicação ao SEO, observou-se que $88,4 \%$ do tráfego orgânico gerado é de usuários que acessam as informações via mecanismos de busca. Na tabela 1 apresenta-se os dados de origem de acesso no período de 01/06/2020 a 31/12/2020. 
Tabela 1 - Usuários por Origem de Acesso: período de 01.06.2020 a 01.12.2020

\begin{tabular}{lcc}
\hline \multicolumn{3}{c}{ Número de Visitas } \\
\hline Origem de Acesso & Total de Usuários & Porcentagem \\
\hline Organic Search & 24.044 & $88,40 \%$ \\
Direct & 2.534 & $9,30 \%$ \\
Referral & 351 & $1,30 \%$ \\
Paid Search & 106 & $0,40 \%$ \\
Social & 88 & $0,30 \%$ \\
Display & 61 & $0,20 \%$ \\
\hline Total & 27184 & $100,00 \%$ \\
\hline
\end{tabular}

Fonte: Elaborado pelos autores (2021), com base no Google Analytcs

A coleta de dados ocorreu durante um período de seis meses, em concreto, de 01-06-2020 a 31-12-2020 e foi comparada com o mesmo período do ano anterior. Na tabela 2 apresenta-se os dados referente ao ano de 2019.

Tabela 2 - Número de visitantes no período de 01.06.2019 a 31.12.2019.

\begin{tabular}{|c|c|c|c|c|c|c|c|c|}
\hline \multicolumn{9}{|c|}{ Número de Visitas } \\
\hline & jun/19 & $\mathrm{jul} / 19$ & ago/19 & set/19 & out/19 & nov/19 & dez/19 & Total \\
\hline Visitas & 1.706 & 1.775 & 1.790 & 1.639 & 1.715 & 1.587 & 1.867 & 12.079 \\
\hline Novos usuários & 1.672 & 1.739 & 1.749 & 1.610 & 1.670 & 1.540 & 1.821 & 11.801 \\
\hline Sessões & 1.860 & 1.990 & 2.072 & 1.830 & 1.830 & 1.737 & 2.028 & 13.397 \\
\hline
\end{tabular}

Fonte: Elaborado pelos autores (2020), com base no Google Analytcs

A mensuração dos resultados da aplicação das técnicas de SEO ocorreu da comparação dos acessos às páginas do site, em dois períodos: De 1 em junho de 2019 a 31 de dezembro de 2019 (Tabela 2), foram contabilizados 23.880 mil acessos ao site e de 1 de em junho de 2020 a 31 de dezembro 2020 (Tabela 3), após a otimização de alguns elementos para os mecanismos de busca, onde o site contabilizou 45.212 mil acessos, obtendo um aumento de visitas em um de total de $89.33 \%$ em um período de 12 meses. 
Tabela 3 - Número de visitantes no período de 01.06.2020 a 31.12.2020.

Número de Visitas

\begin{tabular}{lcccccccc}
\hline & jun/20 & jul/20 & ago/20 & set/20 & out/20 & nov/20 & dez/20 & Total \\
\hline Visitas & 3.092 & 3.546 & 3.505 & 3.566 & 3.674 & 3.503 & 4.077 & 24.963 \\
Novos usuários & 3.005 & 3.450 & 3.410 & 3.444 & 3.563 & 3.377 & 1.3 .961 & 20.249 \\
Sessões & 3.352 & 3.836 & 3.763 & 3.892 & 4.010 & 3.848 & 4.414 & 27.115 \\
\hline
\end{tabular}

Fonte: Elaborado pelos autores (2020), com base no Google Analytcs

Acredita-se que essa evolução tão significativa ocorreu devido à indexação de um maior número de páginas e de conteúdos por parte do Google, tendo isso dado origem a um posicionamento do site na primeira página de resultados de busca.

\section{CONSIDERAÇÕES FINAIS}

Os dados coletados até o momento representam o aspecto qualitativo e o empenho para deixar as páginas do site relevantes para os mecanismos de busca. Ressalta-se que, embora os complexos algoritmos dos mecanismos de busca envolvem diversos fatores, dentre esses fatores o conteúdo informacional, até este momento, vem se mostrando o mais importante. Evidenciou-se, com a análise dos dados, de que o trabalho de SEO, desenvolvido nas páginas do site, aumentou significativamente o interesse do algoritmo de indexação do Google, verificado pelo aumento no número de visitas.

Desse modo, as organizações têm notado a importância dos métodos de SEO para a otimização dos sites nos mecanismos de busca, o que pode impulsionar, no contexto do comércio eletrônico, as visitas, e, consequentemente, as vendas. Sendo assim, o uso desses métodos permite que as organizações alcancem as melhores posições nos resultados orgânicos da pesquisa, independentemente do mercado em que a organização esteja neste ambiente.

Portanto, diante da quantidade de ambientes digitais e de informações na 
Web, o uso das técnicas de SEO, que vem a cada dia inovando a maneira de disponibilização dos conteúdos à sociedade, pode contribuir com a organização, recuperação, acesso e uso da informação de qualquer ambiente digital, viabilizando maior relevância nos resultados de uma busca.

Em suma, o trabalho destaca o conceito de SEO e os resultados positivos de sua aplicação. Para isso, primeiro, o estudo destaca as condições do aparecimento das técnicas com o advento dos grandes atores que promovem mecanismos de busca na internet, oferecendo soluções de organização e recuperação em ambientes descentralizados e distribuídos. Em seguida, destaca a eficiência dessas técnicas usando os relatórios gerenciais oferecidos por esses atores, apresentando alguns resultados parciais do trabalho.

Ressalta-se a importância do profissional da informação como um indutor das técnicas de SEO nos ambientes colaborativos, indicando um campo legítimo de atuação. Sugere-se, portanto, como continuação do trabalho, um aprofundamento prático e um tutorial dessas técnicas, tendo o profissional da informação como interlocutor.

\section{REFERÊNCIAS}

ALMEIDA, G. B. C.; SOUSA, A. K. N.; OLIVEIRA, H. P. C. Arquitetura da informação no contexto de gênero: uma análise do Website da Secretaria Especial de Políticas para Mulheres. Ciência da Informação em Revista, Maceió, v. 5, n. 3, p. 30-42, 2018. Disponível em: https://brapci.inf.br/index.php/res/v/109148. Acesso em: 8 out. 2021.

BATTELLE, J. A busca: como o Google e seus competidores reinventaram os negócios e estão transformando nossas vidas. Rio de Janeiro: Campus, 2005.

BOHÓRQUEZ, I. E. T. Visibilidad Web de las Bibliotecas Públicas Colombianas. Memorias de Bibliotic, 2012. Disponível em: https://silo.tips/download/visibilidad-Web-de-las-bibliotecas-publicascolombianas\#. Acesso em: 8 out. 2021.

CENDÓN, B. V. Ferramentas de busca na Web. Ciência da Informação, Brasília, v. 30, n. 1, p. 39-49, abr., 2001. Disponível em: http://revista.ibict.br/ciinf/article/view/937.

E-BIT. WEBSHOPPERS. 42. ed. 2020. Disponível em: https://www.ebit.com.br/webshoppers. Acesso em: 28 set. 2021. 
GABRIEL, M. Você, eu e os robôs: pequeno manual do mundo digital. São Paulo: Atlas, 2018.

GABRIEL, M.; KISO, R. Marketing na Era Digital: Conceitos, Plataformas e Estratégias. 2. ed. [S.I.]: Atlas, 2020.

Internet Live Stats. Google Search Statistics. Disponível em: https://www.Internetlivestats.com/google-search-statistics/\#share. Acesso em: 23 jun. 2021.

KEMP, S. Digital 2021 Brazil: all the data, trends, and insights you need to help you understand how people use the Internet, mobile, social media, and ecommerce. 2021. Digital in Brazil. Disponível em: https://datareportal.com/reports/digital-2021-brazil. Acesso em: 15 jun. 2021.

LEDFORD, J. L. SEO: Search Engine Optimization Bible. [S.I.]: John Wiley \& Sons, 2007.

MARTINS, D.; CARVALHO JUNIOR, J. M. C. Memória como prática na cultura digital. In: NÚCLEO DE INFORMAÇÃO E COORDENAÇÃO DO PONTO BR. Pesquisa sobre o uso das tecnologias de informação e comunicação nos equipamentos culturais brasileiros: TIC Cultura 2016. 1. ed. São Paulo: Comitê Gestor da Internet no Brasil, 2017. Disponível em: https://cetic.br/media/docs/publicacoes/1/tic_cultura_2018_livro_eletronico.pdf. Acesso em: 20 jun. 2021.

NOVAES. V. E-commerce brasileiro tem alta de $26 \%$ no primeiro trimestre. Economia e Política/Portal Panrotas. 2020. Disponível em: https://www.panrotas.com.br/mercado/economia-e-politica/2020/04/ecommerce-brasileiro-tem-alta-de-26-no-primeiro-trimestre_172823.html. Acesso em: 24 set. 2020.

TU, H. C.; HSIANG, J. An architecture and category knowledge for intelligent information retrieval agents. In: HAWAII INTERNATIONAL CONFERENCE ON SYSTEM SCIENCES, 31., 1998. Proceedings [...]. IEEE, 1998.

ZLATIN, G. On-page vs. Off-page SEO: What's the Difference? Digital Third Coast. 2017. Digital Third Coast. Disponível em: https://www.digitalthirdcoast.com/blog/on-page-off-page-seo-difference. Acesso em: 1 mar. 2021.

\section{SEARCH ENGINE OPTIMIZATION (SEO) APPLIED TO E- COMMERCE}




\begin{abstract}
Objective: This article aims to present the experience of an e-commerce during the implementation of some Search Engine Optimization (SEO) techniques. Methodology: This is a qualitative exploratory study of applied nature, its scope being direct observation, without participants, and having netnography as method for data collection and analysis. Results: It was verified that the use of Search Engine Optimization techniques in the construction of e-commerce sites is efficient and appropriate for the optimization of these environments. Conclusions: We conclude that the Search Engine Optimization techniques and their elements, properly applied, help in the organization, representation and retrieval of information, and make the site more visible in search engines.
\end{abstract}

Descriptors: Search Engine Optimization. E-commerce. Information Retrieval. Web. Search engines.

\title{
OPTIMIZACIÓN DE MOTORES DE BÚSQUEDA (SEO) APLICADA AL COMERCIO ELECTRÓNICO
}

\section{RESUMEN}

Objetivo: Este artículo tiene como objetivo presentar la experiencia de un comercio electrónico durante la aplicación de algunas técnicas de optimización de motores de búsqueda (SEO). Metodología: Se trata de un estudio exploratorio cualitativo de carácter aplicado, siendo su alcance la observación directa, sin participantes, y teniendo como método de recogida y análisis de datos la netnografía. Resultados: Se verificó que el uso de técnicas de Search Engine Optimization en la construcción de sitios de comercio electrónico es eficiente y adecuado para la optimización de estos ambientes. Conclusiones: Se concluye que las técnicas de Search Engine Optimization y sus elementos, debidamente aplicados, ayudan en la organización, representación y recuperación de la información, y hacen que el sitio sea más visible en los motores de búsqueda.

Descriptores: Posicionamiento en buscadores. Comercio electrónico. Recuperación de información. Web. Motores de búsqueda.

Recebido em: 14.10 .2021

Aceito em: 27.12.2021 